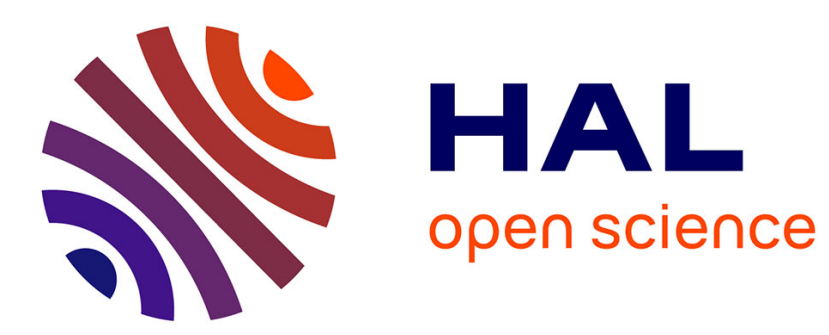

\title{
La promenade sensorielle comme outil pédagogique
}

Marie-Luce Gélard, Olivier Gosselain, Laurent Legrain

\section{To cite this version:}

Marie-Luce Gélard, Olivier Gosselain, Laurent Legrain. La promenade sensorielle comme outil pédagogique. Hermès, La Revue - Cognition, communication, politique, 2016, 74, pp.158-167. halshs03153885

\section{HAL Id: halshs-03153885 \\ https://shs.hal.science/halshs-03153885}

Submitted on 26 Feb 2021

HAL is a multi-disciplinary open access archive for the deposit and dissemination of scientific research documents, whether they are published or not. The documents may come from teaching and research institutions in France or abroad, or from public or private research centers.
L'archive ouverte pluridisciplinaire HAL, est destinée au dépôt et à la diffusion de documents scientifiques de niveau recherche, publiés ou non, émanant des établissements d'enseignement et de recherche français ou étrangers, des laboratoires publics ou privés. 


\section{LA PROMENADE SENSORIELLE COMME OUTIL PÉDAGOGIQUE}

\section{Marie-Luce Gélard, Olivier P. Gosselain, Laurent Legrain}

C.N.R.S. Editions | "Hermès, La Revue »

2016/1 n $74 \mid$ pages 158 à 167

ISSN 0767-9513

ISBN 9782271090171

Article disponible en ligne à l'adresse :

https://www.cairn.info/revue-hermes-la-revue-2016-1-page-158.htm

Distribution électronique Cairn.info pour C.N.R.S. Editions.

(C) C.N.R.S. Editions. Tous droits réservés pour tous pays.

La reproduction ou représentation de cet article, notamment par photocopie, n'est autorisée que dans les limites des conditions générales d'utilisation du site ou, le cas échéant, des conditions générales de la licence souscrite par votre établissement. Toute autre reproduction ou représentation, en tout ou partie, sous quelque forme et de quelque manière que ce soit, est interdite sauf accord préalable et écrit de l'éditeur, en dehors des cas prévus par la législation en vigueur en France. Il est précisé que son stockage dans une base de données est également interdit. 


\section{Marie-Luce Gélard}

Institut universitaire de France - Université

Paris Descartes

Olivier P. Gosselain

Laurent Legrain

Université libre de Bruxelles

\section{La promenade sensorielle comme outil pédagogique}

Cet article a pour ambition de relater une expérience pédagogique croisée autour d'un dispositif commun - une promenade sensorielle en milieu urbain - développée à Paris (université Paris Descartes) et à Bruxelles (Université libre de Bruxelles, ULB). Simple en apparence, ce dispositif n'a cessé de nous surprendre et a engendré une réflexion graduellement plus approfondie sur les méthodes pédagogiques. Issu d'une vague intuition, l'expérience s'est ainsi muée en un outil d'apprentissage et de recherche étonnement fécond, dont les implications ne cessent d'évoluer et de se ramifier.

Ce texte propose d'illustrer concrètement la manière dont les deux expériences ont été menées : leur contexte dans des cursus universitaires spécifiques et leurs retombées pédagogiques. On verra qu'elles se démarquent de l'habituelle absorption passive d'informations par une mise en exergue de l'expérience du monde, mais qu'elles exigent en retour une grande disponibilité des enseignants et des étudiants.

\section{Promenades parisiennes}

L'expérience pédagogique menée avec des étudiants de l'université Paris Descartes en sciences humaines et sociales s'est faite dans le cadre d'un atelier doctoral. Il s'agissait, par le biais de mini-sessions de terrain réalisées sous la forme de "promenades sensorielles", de mettre en avant l'importance des sens et d'une attention portée à ces derniers lors d'enquêtes de terrain ethnologiques et/ou sociologiques.

C'est dans le cadre de recherches en anthropologie des sens que Marie-Luce Gélard suggère une méthode de recueil de données autour de l'anthropologie sensorielle et des ethnographies qu'elle sous-tend. Comment les sens - le plus souvent ignorés alors qu'ils sont des clés de compréhension majeure des sociétés - peuvent-ils devenir des outils d'analyse précieux? Les sens permettent de révéler la manière dont les groupes sociaux utilisent les aspects 
sensoriels comme moyen de communication, d'expression et de manière d'être au monde.

L'idée de proposer des "promenades sensorielles» à des étudiants inscrits en doctorat en sciences humaines et sociales a été suggérée par Olivier Gosselain lors d'un colloque - Rencontres sensorielles/Sensory Meetings : les sens et les sciences sociales - organisé par les universités de Liège et de Bruxelles en 2010. Il présentait cette expérience de recherche qu'il conduit depuis plusieurs années, et j'ai trouvé que cette expérience in situ - que l'on pouvait réaliser n'importe où - pouvait s'avérer être un instrument pédagogique très utile.

Je n'ai pas proprement choisi de réaliser des "promenades sensorielles» avec une visée analytique spécifique, mais plutôt de réaliser de courtes sessions de terrain, par ces promenades sensorielles, afin d'illustrer la manière et l'intérêt d'ethnographier les sens. Le cadre de cet enseignement qui sort des pédagogies habituelles est alors très fécond dans la mesure où il propose aux étudiants de vivre l'expérience en acte et en action.

Dans cet atelier, les étudiants sont amenés à présenter, au sein de leurs propres recherches, les aspects sensoriels et leurs implications tant méthodologiques qu'analytiques. Très généralement, les études proposées semblent éloignées des questions sensorielles et les "promenades" deviennent des révélateurs méthodologiques importants. Ils permettent de développer des axes méthodologiques communs aux recherches en sciences sociales autour d'une ethnographie des sens. La sociologie, l'histoire et l'anthropologie peuvent en effet utiliser ces manifestations sensorielles comme moyen de connaissance de l'autre.

Les expériences que j’ai réalisées ont toutes été conduites sur un parcours parisien spécifique : celui de la «coulée verte». Autrement nommée «promenade plantée» elle occupe l'emplacement de l'ancienne ligne de chemin de fer reliant la place de la Bastille à la Varenne-Saint-Maur.

Ce lieu permet de réaliser une véritable promenade, à l'abri de la circulation tout en restant très en lien avec la ville, bruits, odeurs, luminosité, puisque le parcours, dans son début, surplombe les artères du $12^{\mathrm{e}}$ arrondissement. C'est aussi l'expérience de la nature dans la ville puisqu'il s'agit de jardins plantés d'essences nombreuses, de bassins d'eau, de tunnels et de cascades. On y relève la présence de nombreux oiseaux.

Dans une première phase de l'expérience, il est choisi de marcher depuis le départ du parcours (rue de Lyon), en se concentrant sur les sons durant une vingtaine de minutes. Une courte pause permet ensuite de verbaliser les impressions sonores : les sons entendus diffèrent fréquemment selon les individus, l'expérience illustre déjà des appréciations sensorielles distinctes.

Dans la suite du parcours, je propose de refaire l'expérience de la marche en se concentrant cette fois sur la vue, puis sur l'odorat et enfin sur le toucher : aspérité du sol et revêtement différent (ciment et bois alternent au long de la promenade). Les expériences sensorielles sont ensuite évoquées et verbalisées dans des temps d'arrêt et de partage de la pratique. Les étudiants évoquent le plus souvent la difficulté qu'il y a à conduire cette expérience de dissociation sensorielle et la fatigue ressentie du fait de la concentration nécessaire - le choix de proposer une attention spécifique à chacun des sens successivement ayant pour finalité de les révéler à la conscience individuelle en contraignant le corps à les ressentir.

Lobjectif est alors partiellement atteint : illustrer l'omniprésence des sens et leur interdépendance. C'est aussi l'occasion de montrer que les sens sont dans le quotidien en quelque sorte "dormants». C'est d'ailleurs plus le niveau de conscience qui n'est pas continu, car l'on ne prête pas attention aux sens. La promenade sensorielle révèle leur importance dissimulée dans des automatismes.

Faire cette expérience de concentration sur les sensations (auditives et olfactives) permet de comprendre comment les sens renseignent sur un espace environnant. Les sons permettent de cartographier l'espace connu, dans 
le cadre de terrains ethnographiques. Ils peuvent devenir des moyens d'accès et de compréhension des lieux. Le travail ethnographique de description est alors rendu plus fécond : il ne s'agit plus seulement de décrire visuellement un espace, mais de le ressentir, de le comprendre en quelque sorte plus intensément et, ainsi, de mieux le transcrire verbalement.

$\mathrm{La}$ «promenade sensorielle» invite les étudiants à une considération nouvelle, celle de la place des sens dans toute ethnographie. L'expérience montre ainsi toute la palette des manières d'aborder un terrain de recherche : ces facultés sensorielles deviennent des outils méthodologiques utiles. Les sens sont aussi un moyen de ne plus seulement se focaliser sur des expressions verbales. Le recueil de discours est souvent moins orienté autour du ressenti, même s'il peut l'être parfois. Les entretiens ont souvent pour les étudiants un côté rassurant, car ils semblent donner de la matière, du contenu, des données à un terrain parfois difficile à appréhender; or, ils sont aussi l'expression d'une parole souvent très normée sur un sujet particulier.

Ainsi, les promenades sensorielles ouvrent d'autres horizons d'appréhension du monde environnant, qui serait alors davantage ressenti que verbalisé.

\section{Ballade sensorielle à l'ULB}

L'initiative est menée depuis 2007 au sein d'un enseignement destiné à des étudiants de master en histoire de l'art et archéologie, anthropologie, sociologie, arts du spectacle vivant et géographie. Il s'agit d'une promenade de deux heures environ le long d'un parcours fixe à Bruxelles (depuis le quartier européen jusqu'à la place Royale, en passant par la porte de Namur) durant laquelle les étudiants sont invités à mettre leur corps «à l'écoute de l'environnement», à le «ressentir» en mobilisant tous leurs sens. Les accompagnateurs jouent simplement le rôle de guides et s'efforcent de rester aussi muets et discrets que possible. Dans les jours qui suivent, les étudiants remettent un compte rendu de deux ou trois pages dans lequel ils relatent leur expérience du point de vue des sensations et des émotions éprouvées. Le contenu de ces comptes rendus nourrit les discussions ultérieures sur les possibilités d'investigation par le corps et permet aussi bien d'esquisser le plan de cours que d'en évoquer les enjeux.

\section{Rétroactes}

L'initiative a émergé suite à intuition dans un contexte de questionnements et d'insatisfaction quant à l'enseignement universitaire. L'un d'entre nous (Olivier Gosselain) créait alors un cours de Master destiné aux archéologues et historiens de l'art, dont la thématique concernait le corps, les techniques et les objets. Il n'y avait pas de plan prédéterminé, pas de cahier des charges, juste une envie d'explorer et de partager des connaissances autour d'un champ encore un peu marginal des sciences humaines et sociales.

L’aridité jargonnante de bien des travaux consacrés au corps et à la culture matérielle était apparue d'emblée comme un obstacle. Comment éviter de tomber dans ce piège consistant à discourir sur le «corps» et le «monde matériel» comme s'il s'agissait de concepts abstraits et non de réalités incarnées, soumises à de multiples forces et imposant une approche fondée sur l'expérience sensible (voir la critique féroce que fait Ingold [2007] de cette dérive)? Quelques travaux échappaient néanmoins à ce que Warnier (2009, p. 173-175) qualifie à juste titre «d'effet Magritte» en anthropologie du corps. C'est le cas des contributions du groupe Matière-à-Penser qui montrent bien comment le corps-à-corps avec la matière engendre des sensations, des états, des transformations et des émotions qui retentissent non seulement sur la conscience de soi, mais aussi sur la façon d'appréhender et de penser le monde environnant (Jeanjean, 2006; Julien et Warnier, 
1999). Cette direction semblait incontestablement féconde sur le plan de la recherche, mais comment la transposer à l'enseignement?

Il aurait été pour le moins paradoxal qu'un enseignement centré sur le corps-à-corps avec la matière prenne uniquement la forme d'un cours ex cathedra... C'est pourtant cette forme canonique qui paraît constituer l'horizon d'attente des enseignants et des étudiants en sciences humaines et sociales. Une expérience personnelle des pédagogies actives dans le parcours scolaire, combinée à la conviction qu'il fallait rompre - au moins temporairement - avec le cadre académique classique apporta un début de solution : on sortirait de l'amphithéâtre et on mènerait ensemble une activité centrée sur la sensorialité. Inspiré par la lecture d'articles de Peirce Lewis (1993) - un professeur de géographie qui enseigne la lecture critique du paysage urbain en baladant ses étudiants dans une petite ville - et de Tim Ingold (1999) sur la marche, le projet se précisa : l'activité prendrait la forme d'une déambulation durant laquelle l'environnement urbain ne serait pas appréhendé par la vue seulement, mais par tous les sens. La balade sensorielle était née. Restait à lui trouver un parcours.

C'est en discutant avec un ami géographe - JeanMichel Decroly - qu'une solution fut trouvée. Il venait d'élaborer un parcours touristique dans le quartier des institutions européennes à Bruxelles, à la demande des autorités régionales (Decroly, 2007). Comme il l'explique :

j'avais été amené à discuter avec différents acteurs impliqués dans la conception du schéma directeur du quartier européen. J'avais surtout longuement arpenté le quartier, pour faire des repérages. À cette occasion, j'avais été frappé par les contrastes très forts qui caractérisent tant le bâti du quartier, les fonctions qui s'y déploient, que l'aménagement de l'espace public. Ce qui m'était longtemps apparu comme un quartier uniforme se révélait beaucoup plus divers que prévu. Il est vrai que pour ne pas lasser les visiteurs, nous cherchions des «points de respiration» dans le parcours. Il en a résulté une attention pour les espaces verts ainsi que pour les espaces non marqués par la présence obsédante de l'Europe. C'est donc assez logiquement que, lorsque tu m'as demandé de concevoir une promenade, j'ai jeté mon dévolu sur le quartier européen. Je pense de surcroît qu'il y a peu d'endroits à Bruxelles qui offrent des contrastes si marqués sur des distances courtes (courriel à Olivier Gosselain, 18 déc. 2015).

La première balade sensorielle eut lieu en novembre $2007^{1}$, vers la fin du semestre d'enseignement. Elle fut suivie d'une séance de débriefing la semaine suivante durant laquelle les étudiants évoquèrent ce qu'ils avaient perçu dans les différents segments du parcours et tentèrent, avec l'aide des enseignants, de caractériser les espaces sous l'angle de la sensorialité. Il y avait incontestablement un potentiel pédagogique dans cette expérience, mais il restait à trouver la façon de le concrétiser.

\section{Ajustements}

Depuis 2008, la balade sensorielle est placée en début d'enseignement, juste après la séance d'introduction. Elle marque ainsi une rupture délibérée avec le cadre pédagogique auquel sont habitués les étudiants : une invitation à s'engager autrement dans l'activité d'apprentissage. Initialement lancée sans autre instruction que de mettre son corps à l'écoute de l'environnement, la balade est maintenant encadrée par une série de consignes. La principale est de se transformer en "être en alerte», selon les termes du philosophe José Ortega y Gasset. Dans ses Méditations sur la chasse (2006, p. 145), celui-ci oppose la perception du chasseur à celle du simple promeneur : 
Le touriste voit largement les grands espaces, mais son regard glisse, il ne saisit rien, il ne perçoit pas le rôle de chaque ingrédient dans l'architecture dynamique du paysage. Seul le chasseur, en imitant l'alerte perpétuelle de l'animal sauvage, pour qui tout est danger, voit toute chose et chaque chose fonctionnant comme aide ou adversité, traquenard ou abri.

Une telle mise en alerte requiert «une attention avide» au monde environnant (Pollan, 2006, p. 334). Elle impose un travail de perception permettant de prendre conscience d'une palette de sensations plus large que celles que l'on ramène normalement à soi en marchant dans la ville. Pour aider les étudiants, nous insistons sur la nécessité de dépasser la seule perception visuelle : tous les autres sens doivent être mobilisés. Nous rappelons également qu'il faut aller au-delà des évidences dans l'usage des sens. Le toucher, par exemple, tend à être envisagé comme une activité exploratoire de l'extrémité des doigts. Or, on touche avec bien d'autres parties du corps - notamment en ressentant le froid ou la chaleur, l'humidité, le vent, etc. Et les pieds occupent forcément une place centrale parmi les organes tactiles lors d'une promenade. Ces remarques générales prennent rapidement une forme concrète dans la balade, en raison de ruptures très marquées dans les ambiances urbaines en début de parcours. Généralement inquiets quant à leurs capacités à se servir du corps comme outil d'investigation et parfois très dubitatifs par rapport aux finalités de l'exercice, les étudiants trouvent dans ces ruptures d'ambiance des «prises sensorielles» qui les aident à s'immerger dans l'exercice. C'est pour cette raison que le trajet n'a pas été modifié depuis 2007.

Après quelques hésitations, nous avons fini par accepter la prise de notes ou l'enregistrement vocal durant la balade pour faciliter la rédaction du compte rendu. De nombreux participants prennent également des photos, même si nous les mettons en garde contre le réductionnisme perceptif des images. Par contre, nous les dissuadons de bavarder (sans grand succès pour certains!) ou de faire usage d'éléments susceptibles d'altérer leurs perceptions (baladeurs, téléphones, cigarettes, etc.). Comme la balade se déroule une fois dans l'année, dans le créneau horaire du cours, nous sommes obligés de répartir les étudiants en plusieurs groupes qui se succèdent sur le parcours. Les groupes restent néanmoins rarement inférieurs à une vingtaine de personnes ${ }^{2}$. Ces précisions sont importantes, car elles soulignent le caractère artificiel de l'expérience à laquelle nous convions les étudiants. Loin d'une invitation romantique à renouer avec une corporalité "première» ou "essentielle», la balade sensorielle est un dispositif construit à des fins essentiellement pédagogiques - sur lesquelles nous reviendrons.

L'un des ajustements les plus notables au fil des années concerne notre rapport réflexif et pédagogique à l'exercice. Nous avons dit plus haut que la balade relevait d'une vague intuition et avait pour principal objectif de rompre avec le modus operandi des enseignements en sciences humaines et sociales. Tant que le cours auquel elle était associée restait optionnel, le bouche-à-oreille garantissait une audience intéressée par ce type d'expérience et prête à jouer le jeu. Une succession de réformes font que le cours est à présent obligatoire pour la majorité des étudiants qui le suivent et que tous ne sont pas prêts à se laisser facilement entraîner dans l'exercice. Face à des résistances plus ou moins explicites, nous avons été amenés - sans trop nous en rendre compte au départ - à entreprendre un travail de rationalisation. Celui-ci a notamment consisté à confronter notre balade sensorielle à d'autres expériences du même type (par exemple, Beaufils, 2010) ou à des recherches sur les ambiances urbaines telles que les mènent les membres du Cresson (par exemple, Thibaut et Duarte, 2013), dont nous ignorions tout. Cette confrontation a révélé une différence fondamentale dans les approches : là où nous appréhendons l'expérience via les sensations et les émotions des participants, d'autres développent une 
démarche de type éthologique, approchant de l'extérieur les états intérieurs du piéton, par observation d'une série d'indices corporels (Thomas, 2004). Nous avons également élargi notre perspective au champ de l'art - particulièrement fécond dans le domaine de la marche en ville (par exemple, Davila, 2002; Godfrey et al., 2010) - en enrichissant les discussions par des exemples de propositions artistiques qui explorent non seulement des thématiques similaires aux nôtres, mais poussent parfois la réflexion beaucoup plus loin.

\section{Des premiers résultats aux questionnements}

Plus fondamentalement, nous avons pris à bras-lecorps le matériel contenu dans les centaines de comptes rendus récoltés depuis 2007. Initié en vue de contributions à des colloques sur la ville ou la sensorialité, ce travail analytique a trouvé sa place dans l'enseignement, sous forme d'une séance de cours durant laquelle nous présentons le contenu des comptes rendus de l'année et évoquons certaines observations faites les années précédentes. La séance permet de forger un répertoire commun au groupe, auquel nous faisons régulièrement référence dans les séances ultérieures.

Quoique le contenu puisse changer d'une année à l'autre, quelques constats sont invariablement faits. D'une part, la mobilisation des sens est très asymétrique. Sans surprise, la vue et l'ouïe prédominent, l'odorat n'est évoqué qu'en relation avec certains endroits (saturés d'odeurs nauséabondes ou de nourriture) et le toucher reste tout à fait marginal et plus souvent de l'ordre du surgissement intempestif. Nous retrouvons ici une hiérarchie des sens issue d'une longue sédimentation socio-historique (par exemple Corbin, 1986; Sennett, 2009). D’autre part, nous relevons des sensations et des émotions largement partagées par les participants de la promenade - que nous nommons «consonances». Pour les évoquer, le principal support est une présentation PowerPoint dont les diapositives mettent en série six lieux phares du parcours. Chaque lieu est représenté par une photo en dessous de laquelle sont listés des mots-clés extraits des comptes rendus (nous ne sommons pas les étudiants de classer leurs sensations et émotions par lieu, mais ils le font spontanément). Empruntant une artère très passante du quartier européen, par exemple, les étudiants évoquent majoritairement «bruits de moteur», "cacophonie», "brouhaha», "odeurs de gaz d'échappement», "chaos de la circulation», «architecture gigantesque», «oppression», "vertige», «tension", " écrasement», "vitesse», etc. À l'inverse, le passage dans un parc les conduira à évoquer "couleurs vertes», "silence», "chant des oiseaux», «odeur de terre», «calme», «apaisement», «détente», «rêverie», «bienêtre», etc. Ce phénomène de consonance est discuté avec eux afin de dégager plusieurs notions qui seront explorées dans les leçons suivantes. Au-delà d'un appareil sensoriel commun - le corps humain -, nous évoquons notamment le rôle des expériences et représentations antérieures dans la perception des environnements (que sommes-nous prédisposés à ressentir au milieu de la circulation urbaine ou dans un parc?), la capacité des « saillances perceptuelles » à induire des sensations communes (par exemple, ouverture ou fermeture du champ visuel ou origine et variation des intensités lumineuses pour la vue; mouvement ou absence de mouvement pour l'ouïe), ainsi que l'effet de renforcement des saillances engendré par les ruptures d'ambiances dans le déroulement de la balade.

Mais nous relevons aussi une série de "dissonances» dans les comptes rendus, certes minoritaires, mais suffisamment régulières pour retenir l'attention. Par rapport à une artère passante, un-e étudiant-e évoquera, par exemple, une "sensation de sérénité», "un plaisir esthétique», "une odeur agréable de parfum», "un bruit de rire», tandis qu'un parc sera évoqué comme "triste et sombre», «froid», "désagréable», «de l'ordre de l'illusion", "peu accueillant», "bruyant», etc. Plus difficiles à 
interpréter, ces dissonances nous ont entraînés dans des directions de recherche imprévues - encore largement en chantier - et ont retenti fortement sur le contenu des enseignements.

On peut comprendre les dissonances comme un effet de contingences diverses. Leur entêtement nous oblige à ne pas perdre de vue l'empire des circonstances dans notre travail d'analyse. Chaque étudiant participe à la balade sensorielle avec un bagage différent qui lui vient de ses expériences antérieures. Le travail à accomplir pour comprendre la nature et le fonctionnement de cette capacité de rétention reste immense, mais il est certain que notre degré de familiarité avec un lieu, par exemple, joue un rôle crucial dans les grandes perceptions qui émergeront des micro-changements perceptuels générés par la balade. Nous pensons néanmoins qu'il ne faut pas surévaluer l'action de cet être d'hier qui vit en nous. Les dissonances montrent en effet qu'on ne surestimera jamais le «sporadisme» de la condition humaine (Jankélévitch, 1981). Ce sporadisme consacre l'influence de motifs aussi simples (mais certainement pas triviaux) que l'habitude de la marche et la fatigue éprouvée, la faim ${ }^{3}$, l'état d'esprit du moment, le crédit que l'on accorde à l'exercice, la légitimité des accompagnateurs, la volonté de comprendre leur enthousiasme, l'attitude des autres membres du groupe, l'adéquation de l'équipement vestimentaire (aux conditions météorologiques, à l'activité de la marche). Le dépouillement des multiples comptes rendus de balades sensorielles nous permet aussi de démontrer l'influence de la temporalité des environnements traversés. Les heures de pointe, l'encadrement policier du quartier européen, la pluie ou la trouée dans les nuages qui laisse filtrer le soleil d'octobre, l'amoncellement des poubelles qui attendent la collecte, les effluves des restaurants : tout cela joue sur ce qui, au final, sera noté dans le compte rendu.

En 2015, un petit écart dans nos conventions nous a fait prendre conscience d'un élément qui était passé jusque-là entre les mailles du filet analytique. Une accompagnatrice s'est trompée dans le trajet de la balade et a pénétré dans un parc par une entrée située en contrebas, "contraignant» ainsi son groupe à monter une pente herbeuse que les intempéries des jours précédents avaient rendue glissante. Alors qu'une majorité écrasante d'étudiants qualifie ce parc de «havre de paix», les comptes rendus de ce groupe «égaré» avaient une haute teneur négative. Cette constatation nous a laissés bouche bée, jusqu'à réaliser l'erreur de parcours. Nous avons dès lors été amenés à reconsidérer un point aveugle dans le mode de présentation du contenu des comptes rendus. Avant d'y revenir, soulignons que l'exercice de la balade - bien qu'il se structure d'année en année - ne cesse de révéler des points aveugles. C'est en cela qu'il est pour nous (et pour nos étudiants) un redoutable outil de travail. Comme tout outil réflexif, il engendre une bonne dose de remise en cause.

\section{Des points aveugles qui relancent la réflexion}

Il est clair que certains lieux sont discrétisés par notre attitude lors de la balade. Nous nous y arrêtons et laissons les étudiants s'égailler avant de les rassembler et de continuer notre route. Parfois ces endroits sont physiquement peu délimités, mais ils apparaissent sur le compte rendu comme des endroits discrets du fait même de notre pause/ rassemblement au moment de la balade. L'erreur de parcours qui entraîne la recatégorisation du parc en lieu à haute teneur négative montre avec beaucoup d'éloquence que c'est le trajet, la déclivité, la sensation physique d'un effort bref mais intense qui a joué. La présentation de la balade en une succession de lieux discrets nous fait perdre ces informations sur les impressions de la déambulation elle-même et convie l'idée que sensations et émotions sont contenues dans des lieux spécifiques. Parallèlement, la saisie perceptuelle du contraste entre deux lieux est aussi une information qui se perd dans notre présentation. Il nous faudra trouver des manières d'y remédier. 
Des points aveugles peuvent également prendre la forme d'une absence dans les comptes rendus. L'occultation presque totale de notre propre participation à la création d'environnements sensoriels en est une illustration : le bruit de nos pas ${ }^{4}$, la vision de notre propre corps en train de se mouvoir, sans parler des odeurs que nous portons. Tout se passe comme si l'état d'alerte que nous cherchions à susciter nous transformait en capteurs de sensorialité et non en coproducteurs d'environnements sensoriels saturés. La balade sensorielle est l'occasion de discuter cette conception classiquement passive de la perception. Comme le disait Merleau-Ponty, nous ne sommes pas des claviers dont les touches seraient pressées par les stimuli sensoriels d'un environnement extérieur. S'il fallait garder la métaphore du clavier, il faudrait concevoir un clavier «qui se meut lui-même, de manière à offrir - et selon des rythmes variables - telles de ses notes à l'action en elle-même monotone d'un marteau extérieur» (Varela, 1993, p. 235-236, citant Merleau-Ponty, 1977 [1942]). Parce qu'elle canalise notre déambulation à travers des lieux très contrastés, la trajectoire de la balade sensorielle nous empêche d'affirmer, comme Merleau-Ponty, que l'action de l'environnement est monotone. Mais là n'est pas l'essentiel. L'idée que nous retirons de cette citation est celle d'une coproduction d'environnements chargés de sensorialité, environnements que l'anthropologue Adam Chau (2008) appelle des «sensoriums", par analogie avec les aquariums. Ce rôle actif du processus perceptuel, point aveugle des comptes rendus, nous a conduits à présenter dans la suite du cours des trousses à outils qui invitent à envisager la perception en tant qu'activité située (comme le fait le concept de "prise» des sociologues Bessy et Chateauraynaud, 1995, ainsi que différentes déclinaisons de «l'agentivité»).

Un autre point aveugle devrait prochainement mener à la construction d'une boîte à outils spécifique. Il concerne l'absence d'évocation du substrat corporel des émotions dans les comptes rendus. Les étudiants mobilisent plutôt des étiquettes d'usage commun et constant dans la vie quotidienne, comme se sentir «angoissé», «insécurisé», "oppressé», «joyeux», etc. Il est probable que cette occultation du corps - alors même que nous demandons d'y être attentif - est due à l'hyper-psychologisation de nos sociétés et/ou à l'habitude de manipuler des étiquettes culturelles pour parler du corps (ce «sac à surprises» dont parle Daniel Pennac dans son roman Journal d'un corps). À ce stade, nous ne savons pas comment aborder le sujet avec nos étudiants et leur renvoyons la question sans y apporter de réponse (ce qui est déjà un acte pédagogique à nos yeux). Car repartir d'une balade sensorielle pour enseigner veut aussi dire se faire surprendre par ce que le dépouillement des comptes rendus apporte en termes de données et de thématiques à traiter. Il faut bien parfois poser les limites de ce qui sera travaillé et se contenter de saisir du regard les vastes territoires qui resteront en friche. Ne donne-t-on pas, en procédant de la sorte, une image plus fidèle de ce qu'est la connaissance : un processus de transformation continu (Lave, 2011)?

En tant que dispositif pédagogique, la balade sensorielle est une invitation à la co-construction de la connaissance et à l'engagement participatif. Les enseignants ont tendance à penser que la participation de leurs étudiants émergera naturellement du fait de la motivation qu'enseignant et étudiants partagent, et de la puissance séduisante des notions et concepts qui ont «fait leurs preuves». Mais les choses ne sont pas si simples. Comme tous les dispositifs de co-construction de la connaissance, la balade sensorielle nécessite du temps et des ajustements mutuels pour être effective (dépasser la simple bonne volonté de co-construire) et efficace (porter ses fruits). Si elle n'est pas suivie d'un débriefing qui trouve ses lignes de force analytiques dans la compilation rigoureuse (et donc chronophage) des comptes rendus, compilation elle-même appuyée sur des réflexions et des concepts disponibles dans la littérature, la balade sensorielle n'est qu'une expérience légèrement contraignante dans un cours exotique. Pour certains étudiants, c'est ce qu'elle est et restera. C'est 
ce qu'elle risque également de demeurer, année après année, pour les enseignants qui restent guidés par l'idée d'une connaissance figée et qui ont peu de temps à consacrer aux contributions des étudiants. À Bruxelles, deux semaines s'écoulent entre la balade et le débriefing. Deux semaines durant lesquelles nous tentons de lire tous les comptes rendus et de les compiler. D'y trouver des consonances, des dissonances, des manques. Deux semaines durant lesquelles nous sommes surpris, ici ou là, par des commentaires qui mettent en cause nos manières de compiler,

\section{NOTES}

1. Dans des conditions météorologiques exécrables, qui retentirent inévitablement sur les perceptions des participants.

2. Plus de 80 étudiants prenaient part à l'expérience en 2015 .

3. La fatigue et la faim sont envisagées par une grande majorité des étudiants comme des motifs d'arrêt de l'exercice au beau milieu de la balade. «J'avais trop faim» ou «j'étais trop fatigué» pour continuer à percevoir. Nous défendons l'idée que ces états canalisent l'acte de perception, mais que nous ne pouvons «cesser de

\section{RÉ F ÉRE NCES B I B L I O GR A P H I Q U E S}

BEAUfils, T., «Une fragrance douceâtre de crottin de cheval : balade olfactive à travers la Flandre et les Pays-Bas», Septentrion, $\mathrm{n}^{\circ} 1,2010$, p. 40-45.

Bessy, C. et Chateauraynaud, F., Experts et faussaires. Pour une sociologie de la perception, Paris, Métailié, 1995.

Chau, A. Y., "The Sensorial Production of the Social», Ethnos, vol. 73, n 4, 2008, p. 485-504.

Corbin, A., Le Miasme et la jonquille. L'odorat et l'imaginaire social, XVIII-XIX ${ }^{e}$ siècle, Paris, Flammarion, 1986.

Corbin, A., "Histoire et anthropologie sensorielle», Anthropologie et Sociétés, n 14, 1990, p. 13-24. qui appellent d'autres réflexions ou qui montrent que les concepts et notions que nous avons préparés pour la suite ne suffiront pas pour appréhender et mettre en ordre ce foisonnement. Car la trousse conceptuelle que nous travaillons au fur et à mesure des années sera elle-même travaillée, déformée, démembrée par la richesse des comptes rendus. C'est risqué. Il faut que «ça prenne». On ne s'étonnera pas que nous soyons saisis de trac en commençant le cours, comme l'est le musicien qui monte sur scène en se demandant si le concert sera bon ce soir.

percevoir ». Le sommeil est le niveau le plus bas de la perception, mais il ne signifie pas son arrêt. Ce type de propositions est discuté en séance de débriefing.

4. Les claquements de nos pas reviennent sur le devant de la scène perceptuelle lorsque les étudiants outillent leur perception par l'emploi d'un enregistreur et d'un micro. Ceci fut l'occasion pour nous d'aborder le concept de «médiation» tel qu'il est développé notamment dans les travaux d'Antoine Hennion (1988).

Davila, T., Marcher, créer. Déplacements, flâneries, dérives dans l'art de la fin $d u X X^{e}$ siècle, Paris, éditions du Regard, 2002.

Decroly, J.-M., Ma Capitale, mes quartiers européens, Bruxelles, BITC, 2007.

GÉLARD, M.-L., «L'anthropologie sensorielle en France. Un champ en devenir?», L'Homme, n² 217, 2016, p. 91-108.

Godfrey, M., Biesenbach, K., Greenberg, K. et Snauwaert, D., Francis Alÿs : a Story of Deception, Bruxelles, Wiels - Centre d'art contemporain, 2010.

Hennion, A., Comment la musique vient aux enfants. Une anthropologie de l'enseignement musical, Paris, Anthropos, 1988. 
Howes, D., Empire of Senses. The Sensual Culture Reader, Oxford, Berg, 2004.

Howes, D., «L'esprit multisensoriel, ou la modulation de la perception », Communications, $n^{\circ} 86,2010$, p. 37-46.

INGOLD, T., "Culture on the Ground. The World Perceived Through the Feet», Journal of Material Culture, vol. 9, n 3, 2004, p. 315-340.

IngolD, T., «Materials Against Materiality», Archaeological Dialogues, vol. 14, n 1, 2007, p. 1-16.

JANKÉLÉVITCH, V., Le Je-ne-sais-quoi et le presque-rien. Tome 2 : la méconnaissance - le malentendu, Paris, Seuil, coll. "Point Essais», 1981.

Jeanjean, A., Basses ceuvres, une ethnologie du travail dans les égouts, Paris, éditions du CTHS, 2006.

Julien, M.-P. et Warnier, J.-P. (dir.), Approches de la culture matérielle. Corps à corps avec l'objet, Paris, L'Harmattan, 1999.

LAVE, J., Apprenticeship in Critical Ethnographic Practice, Chicago, University of Chicago Press, 2011.

LEWIS, P., "Common Landscapes as Historic Documents», in LUBAR, S. et Kingery, W. D. (dir.), History From Things. Essays on Material Culture, Washington, Smithsonian Institution, 1993, p. 115-139.
Merleau-Ponty, M., La Structure du comportement, Paris, Presses universitaires de France, 1942.

Ortega y Gasset, J., Méditations sur la chasse, Québec, Septentrion, 2006 [1957].

PennaC, D., Journal d'un corps, Paris, Gallimard, 2012.

Pollan, M., The Omnivore's Dilemma. The Search For a Perfect Meal in a Fast-Food World, Londres, Bloomsbury, 2006.

Sennett, R., La Conscience de l'oeil : urbanisme et société, Paris, Verdier, 2009.

Thibaut, J.-P. et Duarte, C. R. (dir.), Ambiances urbaines en partage : pour une écologie de la ville sensible, Genève, MetisPresse, 2013.

Thomas, R., «Quand le pas fait corps et sens avec l'espace. Aspects sensibles et expressifs de la marche en ville», Cybergeo : European Journal of Geography [en ligne], 2004. En ligne sur : <cybergeo. revues.org/4304>, consulté le 2/2/2016.

Varela, F., Thompson, E. et Rosh, E., L'Inscription corporelle de l'esprit. Sciences cognitive et expérience humaine, Paris, Seuil, coll. «La couleur des idées», 1993.

WARNIER, J.-P., «Le corps du litige en anthropologie», in Memmi, D., Guillo, D. et Martin, O. (dir.), La Tentation du corps, Paris, éditions de l'EHESS, 2009, p. 171-197. 